TRANSACTIONS OF THE

AMERICAN MATHEMATICAL SOCIETY

Volume 359, Number 1, January 2007, Pages 417-426

S 0002-9947(06)03991-2

Article electronically published on August 24, 2006

\title{
ON GENERALIZATIONS OF LAVRENTIEFF'S THEOREM FOR POLISH GROUP ACTIONS
}

\author{
LONGYUN DING AND SU GAO
}

\begin{abstract}
It is shown that for every Polish group $G$ that is not locally compact there is a continuous action $a$ of $G$ on a $\Pi_{1}^{1}$-complete subset $A$ of a Polish space $X$ such that $a$ cannot be extended to any superset of $A$ in $X$. This answers a question posed by Becker and Kechris and shows that an earlier theorem of them is optimal in several aspects.
\end{abstract}

\section{INTRODUCTION}

A classical theorem of Lavrentieff (4]) states that

Let $X$ and $Y$ be completely metrizable spaces. Let $A \subseteq X, B \subseteq Y$, and $h: A \rightarrow B$ a homeomorphism. Then $h$ can be extended to a homeomorphism $h^{*}: A^{*} \rightarrow B^{*}$, where $A \subseteq A^{*}, B \subseteq B^{*}$, and $A^{*}$ and $B^{*}$ are $G_{\delta}$ sets in $X$ and $Y$, respectively.

This theorem has been generalized to various different contexts. One of the generalizations is to replace the single homeomorphism by a group of homeomorphisms in the context that $X=Y$ and $A=B$. Specifically, Becker and Kechris proved the following theorem in [1], Theorem 2.2.7.

Theorem 1.1 (Becker-Kechris [1]). Let $G$ be a Polish group, $X$ a Polish space, $A \subseteq X$ and $a: G \times A \rightarrow A$ a continuous action of $G$ on $A$. Then the action a can be extended to a continuous action $a^{*}: G \times A^{*} \rightarrow A^{*}$, where $A \subseteq A^{*}$ and the following definability conditions for $A^{*}$ hold:

(1) If $G$ is locally compact, then $A^{*}$ can be taken to be a $G_{\delta}$ subset of $X$.

(2) If $G$ is a general Polish group, then $A^{*}$ can be taken to be a $\Pi_{1}^{1}$ subset of $X$.

(3) If $G$ is general but $A$ is analytic, then $A^{*}$ can be taken to be a Borel subset of $X$.

The theorem provides a full generalization of Lavrentieff's theorem in the case $G$ is locally compact Polish. For general Polish group actions, Becker and Kechris asked the following question (Open Problem 2.2.8 of [1]).

Question 1.2 (Becker-Kechris [1). Can the set $A^{*}$ in the above theorem be taken to be $G_{\delta}$ in the case of an arbitrary Polish group?

In this paper we give a negative answer for this question. In fact we prove results much stronger than what we need to construct a counterexample. In addition to

Received by the editors December 13, 2004.

2000 Mathematics Subject Classification. Primary 54H05, 22 F05.

(C)2006 American Mathematical Society Reverts to public domain 28 years from publication 
showing that there are Polish group partial actions which cannot be extended to $G_{\delta}$ sets, we prove that there are such actions which cannot be extended to any analytic sets. This implies that the above theorem of Becker and Kechris is optimal. Moreover, we show that for any Polish group $G$ which is not locally compact there are partial actions of $G$ which are counterexamples of the type described above. Thus the extendability of a partial action to a $G_{\delta}$ set is a characteristic property of all locally compact groups. The main theorem we establish is the following.

Theorem 1.3. Let $G$ be a non-locally-compact Polish group. Then there is a Polish space $X, a \mathbf{\Pi}_{1}^{1}$-complete subset $A$ of $X$ and a continuous action $a: G \times A \rightarrow A$ such that the action a cannot be extended.

In the statement of the theorem the action $a$ cannot be extended to any superset of $A$, namely, the action cannot be extended to even one more point. Such nonextendable sets are the focus of our study in this paper.

The paper is organized as follows. In Section 2 we formulate the concept of nonextendability and give examples of non-extendable sets, thus obtaining counterexamples to Question 1.2. In Section 3 we further characterize the non-extendability property and prove the main results of this paper. In Section 4 we follow up on the concepts studied in the previous sections and give examples and remarks about further research. Throughout the paper we follow the notation in 2$]$ and $[1$. The research of the second author was partially supported by NSF grant DMS-0100439.

\section{CountereXamples AND THE NON-EXtendability PROPERTY}

We first investigate a property of a Polish group action which connects directly to the counterexamples of the question of Becker and Kechris. First recall from [1] that in the case of a Polish group $G$ acting continuously on a Polish space $X$, we call $X$ a Polish $G$-space.

Definition 2.1. Let $G$ be a Polish group and $X$ a Polish $G$-space. $X$ is said to have the non-extendability property if there are subsets $A$ and $Y$ of $X$ such that

(i) $A \subseteq Y$ and $A$ is dense in $Y$,

(ii) $Y$ is $G_{\delta}$ and $A$ is not $G_{\delta}$,

(iii) $A$ is $G$-invariant, and

(iv) for every $x \in Y \backslash A$ there is a $g \in G$ such that $g \cdot x \notin Y$.

In this case we also say that $A$ and $Y$ witness the non-extendability property of the Polish $G$-space $X$.

Spaces with the non-extendability property immediately give counterexamples to Question 1.2.

Lemma 2.2. Let $G$ be a Polish group, $X$ a Polish $G$-space, and $A$ and $Y$ witness the non-extendability property of $X$. Then in the Polish space $Y$ the action of $G$ on A cannot be extended.

Proof. Note that $Y$ is indeed a Polish space since it is a $G_{\delta}$ subset of $X$. Also the action of $G$ on $A$ is well defined since $A$ is $G$-invariant. Let $b: G \times X \rightarrow X$ be the continuous action of $G$ on $X$ and let $a=b \uparrow(G \times A)$. Let $a^{*}: G \times A^{*} \rightarrow A^{*}$ be any extension of $a$ to a set $A^{*}$ satisfying $A \subseteq A^{*} \subseteq \bar{A}$, where $\bar{A}$ is the closure of $A$ in $X$. We claim that $a^{*}=b \uparrow\left(G \times A^{*}\right)$. 
To see this, let $x \in A^{*}$ and $\left\{x_{n}\right\}$ be a sequence of elements of $A$ such that $x_{n} \rightarrow x$ as $n \rightarrow \infty$. Since both $a^{*}$ and $b$ are continuous extensions of $a$, we have that for any $g \in G$,

$$
a^{*}(g, x)=\lim _{n \rightarrow \infty} a^{*}\left(g, x_{n}\right)=\lim _{n \rightarrow \infty} a\left(g, x_{n}\right)=\lim _{n \rightarrow \infty} b\left(g, x_{n}\right)=b(g, x) .
$$

Now condition (iv) of the non-extendability property implies that $a$ cannot be extended. If $A^{*} \subseteq Y$ and $A^{*} \backslash A \neq \emptyset$, then for any $x \in A^{*} \backslash A$ there is $g \in G$ such that $b(g, x) \notin Y$, hence $a^{*}(g, x) \notin Y$, a contradiction.

In the Polish space $Y$ the set $A$ (with the continuous $G$-action) will be called nonextendable. It follows from Theorem 1.1 that any non-extendable $A$ is necessarily $\boldsymbol{\Pi}_{1}^{1}$. Next we deduce that existence of any non-extendable set implies the existence of a $\boldsymbol{\Pi}_{1}^{1}$-complete non-extendable set. Note that in the above proof we did not use the condition that $A$ is not $G_{\delta}$, hence the lemma makes sense also for locally compact groups. For the next result the fact that $A$ is not $G_{\delta}$ will be needed. Before stating the result we need to recall the following notation.

For a topological space $X$ consider the space

$$
K(X)=\{K \subseteq X: K \text { is compact }\}
$$

equipped with the Vietoris topology (see [2, 4.F). If $X$ is Polish, then so is $K(X)$. Now suppose a Polish group $G$ acts continuously on $X$. Then the action naturally induces a continuous $G$-action on $K(X)$ :

$$
g \cdot K=\{g \cdot x: x \in K\}
$$

for $g \in G$ and $K \in K(X)$.

Lemma 2.3. Let $G$ be a Polish group, $X$ a Polish $G$-space, and $A$ and $Y$ witness the non-extendability property of $X$. Then in the Polish space $K(Y)$ the subset $K(A)$ is $\boldsymbol{\Pi}_{1}^{1}$-complete and the action of $G$ on $K(A)$ cannot be extended.

Proof. As remarked above $A$ is $\boldsymbol{\Pi}_{1}^{1}$, from which it follows that $K(A)$ is $\boldsymbol{\Pi}_{1}^{1}$ in $K(X)$. By the Kechris-Louveau-Woodin dichotomy theorem for $\boldsymbol{\Pi}_{1}^{1}, \sigma$-ideals of compact sets ([3] or see [2, 33.C) $K(A)$ are $\Pi_{1}^{1}$-complete in $K(X)$ if $A$ is not $G_{\delta}$ in $X$.

To see that in the Polish space $K(Y)$ the action of $G$ on $K(A)$ cannot be extended, we check that $K(A)$ and $K(Y)$ actually witness the non-extendability property of $K(X)$. It is immediate that $K(A)$ is $G$-invariant since $A$ is $G$-invariant. Also $K(A)$ is dense in $K(Y)$ since $A$ is dense in $Y$. Thus to finish the proof it is enough to check condition (iv) of the non-extendability property. Let $K \in K(Y) \backslash K(A)$. Then there is $x \in K$ so that $x \in Y \backslash A$. By the non-extendability of $X$ there is $g \in G$ such that $g \cdot x \notin Y$, thus $g \cdot K \nsubseteq Y Y$ and therefore $g \cdot K \notin K(Y)$.

Now the lemma follows from the preceding lemma.

Thus in order to get a counterexample to Question 1.2 in a strong sense we only need to produce an example of an action with the non-extendability property. In the next section we will show that it is fairly easy to produce such examples. Namely, as long as the action produces at least one orbit which is not $F_{\sigma}$, it has the non-extendability property. However, for now, we would like to present a less trivial example. This was the first example found to have the non-extendability property. In view of the results of the next section this example is an overkill for Question 1.2. However, we think it is interesting because it arises in a natural context and induces more open questions. 
Let $S_{\infty}$ be the infinite permutation group, i.e., the group of all permutations of $\mathbb{N}$. With the pointwise convergence topology $S_{\infty}$ is a Polish group. Consider the natural action $a$ of $S_{\infty}$ on the product space $\mathbb{R}^{\mathbb{N}}$ given by

$$
a(g, x)=g \cdot x=x \circ g^{-1}
$$

in other words, for all $n \in \mathbb{N}$,

$$
(g \cdot x)(n)=x\left(g^{-1}(n)\right) .
$$

It is easy to see that $a$ is a continuous action.

For $x \in \mathbb{R}^{\mathbb{N}}$ and $n \in \mathbb{N}$, let

$$
\sigma(x)(n)=\sum_{k=0}^{n} x(k) .
$$

Then $\sigma: \mathbb{R}^{\mathbb{N}} \rightarrow \mathbb{R}^{\mathbb{N}}$ is a homeomorphism.

Now we define

$$
\begin{aligned}
Z & =\left\{x \in \mathbb{R}^{\mathbb{N}}: \text { There is a subsequence of } x \text { converging to } 0\right\} \\
& =\left\{x \in \mathbb{R}^{\mathbb{N}}: \forall k \forall m \exists n>m\left(|x(n)|<\frac{1}{k}\right)\right\} .
\end{aligned}
$$

Then $Z$ is a $G_{\delta}$ subset of $\mathbb{R}^{\mathbb{N}}$. Let $Y=\sigma^{-1}(Z)$; then $Y$ is $G_{\delta}$ in $\mathbb{R}^{\mathbb{N}}$, too.

Next define a subset $A \subseteq Y$ by

$$
A=\left\{x \in \mathbb{R}^{\mathbb{N}}: \sum_{n=0}^{\infty} x(n) \text { converges absolutely to } 0\right\} .
$$

Theorem 2.4. The sets $A$ and $Y$ defined above witness the non-extendability property of the Polish $S_{\infty}$-space $\mathbb{R}^{\mathbb{N}}$.

Proof. We check the conditions of the non-extendability property by a number of claims.

Claim 1. Both $A$ and $Y \backslash A$ are dense in $Y$.

For $x \in Y$ and $m \in \mathbb{N}$, let

$$
\begin{aligned}
x_{m}^{A} & =\left(x(0), x(1), \ldots, x(m),-\sum_{k=0}^{m} x(k), 0,0, \ldots, 0, \ldots\right), \\
x_{m}^{Y \backslash A} & =\left(x(0), x(1), \ldots, x(m),-\sum_{k=0}^{m} x(k), 1,-1,1,-1, \ldots\right) .
\end{aligned}
$$

Then $x_{m}^{A} \in A, x_{m}^{Y \backslash A} \in Y \backslash A$, and both $x_{m}^{A} \rightarrow x$ and $x_{m}^{Y \backslash A} \rightarrow x$ as $m \rightarrow \infty$.

Claim 2. $A$ is invariant under the action $a$.

This is an elementary theorem of analysis concerning absolutely convergent series.

Claim 3. $A$ is not $G_{\delta}$ in $Y$.

First note that $A$ is relatively $F_{\sigma}$ in $Y$. To see this, consider the $F_{\sigma}$ subset of $\mathbb{R}^{\mathbb{N}}$

$$
\ell^{1}=\left\{x \in \mathbb{R}^{\mathbb{N}}: \sum_{n=0}^{\infty}|x(n)|<\infty\right\},
$$

and note that $A=\ell^{1} \cap Y$ since absolute convergence implies convergence for a series. 
Now $A$ cannot be $G_{\delta}$ in $Y$, since otherwise both $A$ and $Y \backslash A$ would be $G_{\delta}$ in $Y$, and by Claim 1 both of them would be dense $G_{\delta}$, hence comeager.

To finish our proof, it remains to check condition (iv) of the non-extendability property.

Claim 4. For any $x \in Y \backslash A$ there is a $g \in G$ such that $x \circ g^{-1} \notin Y$.

If $x \in Y \backslash A$, then $x$ is not absolutely convergent. By a well-known theorem of real analysis, the terms of $x$ can be rearranged to form a new series which converges to $+\infty$. That is to say that there is a $g \in S_{\infty}$ such that

$$
\sum_{k=0}^{\infty} x\left(g^{-1}(k)\right)=+\infty
$$

thus $x \circ g^{-1} \notin Y$.

Since the set $A$ in this theorem is $F_{\sigma}$ but not $G_{\delta}$, the $\Pi_{1}^{1}$-completeness of $K(A)$ follows from an old result of Hurewicz (see 2 27.B). In the next section we will show that an $F_{\sigma}$ non-extendable set can always be found, thus one does not need the Kechris-Louveau-Woodin dichtomy theorem to establish the results of this paper. To summarize, we have established the following theorem in this section.

Theorem 2.5. There is a Polish group $G$, a Polish space $X, A \subseteq X$ and a continuous action $a: G \times A \rightarrow A$ of $G$ on $A$ such that the action a cannot be extended to any analytic set.

\section{MAin Results}

In this section we give more transparent characterizations of the non-extendability property and prove Theorem 1.3, a generalization of the result of the last section to arbitrary non-locally-compact Polish groups.

We first recall some notation from [2]. Let $G$ be a Polish group and $X$ a Polish $G$-space. For $M \subseteq X,[M]_{G}$ denotes the saturation of $M$, i.e., the smallest $G$ invariant set containing $M$, and similiarly $(M)_{G}$ the hull of $M$, i.e., the largest $G$-invariant set contained in $M$. In symbols,

$$
[M]_{G}=\{x \in X: \exists g \in G(g \cdot x \in M)\}
$$

and

$$
(M)_{G}=\{x \in X: \forall g \in G(g \cdot x \in M)\} .
$$

It is straightforward to see that for any $M \subseteq X,[X \backslash M]_{G}=X \backslash(M)_{G}$, and for any sequence $M_{n} \subseteq X$,

$$
\left[\bigcup_{n} M_{n}\right]_{G}=\bigcup_{n}\left[M_{n}\right]_{G}
$$

and

$$
\left(\bigcap_{n} M_{n}\right)_{G}=\bigcap_{n}\left(M_{n}\right)_{G} .
$$

If $A$ and $Y$ witness the non-extendability property of $X$, then conditions (iii) and (iv) can be restated simply as $A=(Y)_{G}$. 
We have the following characterization of the non-extendability property.

Theorem 3.1. Let $G$ be a Polish group and $X$ a Polish $G$-space. Then the following are equivalent:

(i) $X$ has the non-extendability property.

(ii) There is a $G_{\delta}$ set $Y$ in $X$ such that $(Y)_{G}$ is not $G_{\delta}$.

(iii) There is an open set $U$ in $X$ such that $(U)_{G}$ is not $G_{\delta}$.

(iv) There is a $F_{\sigma}$ set $M$ in $X$ such that $[M]_{G}$ is not $F_{\sigma}$.

(v) There is a closed set $F$ in $X$ such that $[F]_{G}$ is not $F_{\sigma}$.

Proof. The direction (i) $\Rightarrow$ (ii) is immediate from the remark preceding the theorem. The equivalence of (ii) through (v) follows from the identities concerning saturations and hulls reviewed above. To see that (ii) $\Rightarrow(\mathrm{i})$, let $Y_{0}$ be a $G_{\delta}$ subset of $X$ and $A=\left(Y_{0}\right)_{G}$ which is not $G_{\delta}$. Let $Y=\bar{A} \cap Y_{0}$, where $\bar{A}$ is the closure of $A$ in $X$. Then $Y$ is $G_{\delta}, A \subseteq Y, A$ is dense in $Y$ and $(Y)_{G}=(\bar{A})_{G} \cap\left(Y_{0}\right)_{G}=A$. Thus the sets $A$ and $Y$ witness the non-extendability property of $X$.

The following corollary is immediate.

Corollary 3.2. Let $G$ be a Polish group and $X$ a Polish $G$-space. If $X$ contains an orbit which is not $F_{\sigma}$, then $X$ has the non-extendability property.

Proof. Let $x$ be any element of such an orbit. Then $F=\{x\}$ is closed and $[F]_{G}=$ $[x]_{G}$ is not $F_{\sigma}$, satisfying (v) of the preceding theorem.

If $G$ is a locally compact Polish group, then every orbit of a continuous $G$-action is necessarily $K_{\sigma}$, hence $F_{\sigma}$. On the other hand, if $G$ is not locally compact, we show below that a non- $F_{\sigma}$ orbit can be produced.

In the following proof we will use a left-invariant compatible metric on a Polish group $G$. Recall that a metric $d$ on a Polish group $G$ is left-invariant if for all $g, h, k \in G$,

$$
d(g h, g k)=d(h, k) .
$$

A classicial theorem of Birkhoff and Kakutani states that all metrizable topological groups admit left-invariant compatible metrics (cf. 2], 9.A). Also note that if $d$ is a left-invariant compatible metric on $G$, then the metric $d^{\prime}$ defined by

$$
d^{\prime}(x, y)=\frac{d(x, y)}{1+d(x, y)}
$$

is also a left-invariant compatible metric, but it satisfies that $d^{\prime} \leq 1$.

Theorem 3.3. If $G$ is a non-locally-compact Polish group, then there is a Polish $G$-space $X$ with the non-extendability property.

Proof. Let $d \leq 1$ be a left-invariant compatible metric on $G$. Let $L(G, \mathbb{I})$ denote all the Lipschitz functions from $G$ to the unit interval $\mathbb{I}=[0,1]$ :

$$
L(G, \mathbb{I})=\left\{f: G \rightarrow \mathbb{I}: \forall h_{1}, h_{2} \in G\left|f\left(h_{1}\right)-f\left(h_{2}\right)\right| \leq d\left(h_{1}, h_{2}\right)\right\}
$$

equipped with the pointwise convergence topology. By the Arzela-Ascolli theorem, $L(G, \mathbb{I})$ is a compact metrizable space. Moreover $G$ acts on $L(G, \mathbb{I})$ by $(g \cdot f)(h)=$ $f\left(g^{-1} h\right)$, and the action is continuous. $L(G, \mathbb{I})$ is a Polish $G$-space. 
For any $k \in G$, we can define a Lipschitz function $f_{k}$ on $G$ by $f_{k}(h)=d(h, k)$ for $h \in G$. It is straightforward to check that the map

$$
\begin{aligned}
\tau: G & \rightarrow L(G, \mathbb{I}), \\
k & \mapsto f_{k}
\end{aligned}
$$

is a homeomorphic embedding from $G$ into $L(G, \mathbb{I})$. It is also easy to see that $g \cdot f_{k}=f_{g k}$, since $d$ is left invariant. So $\tau(G)$ is indeed an orbit and is $G_{\delta}$.

Let $X=\overline{\tau(G)}$ be the closure of $\tau(G)$ in $L(G, \mathbb{I})$. Then $X$ is a closed $G$-invariant subset of $L(G, \mathbb{I})$ and hence itself a Polish $G$-space. To verify that $X$ has the nonextendability property, by the preceding corollary it is enough to prove that $\tau(G)$ is not $F_{\sigma}$ in $X$. Note that $X$ is compact and $\tau(G)$ is dense in $X$. Since $G$ is not locally compact, every point in $\tau(G)$ has no compact neighborhood.

For any $k \in G$ and open neighborhood $U$ of $f_{k}$ in $X$, we can find an open neighborhood of $f_{k}$ in $X$, say $V$, such that $\bar{V} \subseteq U$. Then $\bar{V}$ is compact. $\bar{V} \nsubseteq \tau(G)$, since $f_{k}$ has no compact neighborhood in $\tau(G)$. So there is $f \in X \backslash \tau(G)$ such that $f \in \bar{V} \subseteq U$. This shows that $X \backslash \tau(G)$ is dense in $X$.

Now, since $\tau(G)$ and $X \backslash \tau(G)$ are both dense in $X$ and $\tau(G)$ is $G_{\delta}$ in $X$, it follows that $X \backslash \tau(G)$ cannot be $G_{\delta}$ in $X$. Therefore $\tau(G)$ is not $F_{\sigma}$ in $X$.

The following theorems summarize our main results of this section.

Theorem 3.4. Let $G$ be a non-locally-compact Polish group. Then there is a Polish space $X, a \Pi_{1}^{1}$-complete subset $A$ of $X$ and a continuous action $a: G \times A \rightarrow A$ such that the action a cannot be extended.

Proof. Let $X_{0}$ be the space constructed in the preceding proof with the nonextendability property. Let $A_{0}$ and $Y_{0}$ be any witness for the non-extendability property of $X_{0}$. Then let $X=K\left(X_{0}\right), Y=K\left(Y_{0}\right)$ and $A=K\left(A_{0}\right)$, and let the action $a: G \times A \rightarrow A$ be the induced action of $G$ on $A$. By Lemma $2.3 A$ is $\boldsymbol{\Pi}_{1}^{1}$-complete and the action $a$ cannot be extended.

Theorem 3.5. Let $G$ be a Polish group. The following are equivalent:

(a) $G$ is locally compact.

(b) In every Polish $G$-space every orbit is $K_{\sigma}$.

(c) In every Polish $G$-space every orbit is $F_{\sigma}$.

(d) In every Polish $G$-space the non-extendability property fails.

(e) In every Polish $G$-space the saturation of a closed set is $F_{\sigma}$.

(f) In every Polish $G$-space the saturation of an $F_{\sigma}$ set is $F_{\sigma}$.

(g) In every Polish $G$-space the saturation of a compact set is $K_{\sigma}$.

(h) In every Polish $G$-space the saturation of a $K_{\sigma}$ set is $K_{\sigma}$.

(i) For any Polish space $X$, any subset $A$ and continuous action $a: G \times A \rightarrow A$, the action a can be extended to a continuous action on a $G_{\delta}$ subset of $X$.

Proof. The directions (a) $\Rightarrow(\mathrm{b}) \Rightarrow(\mathrm{c})$ are immediate. (c) $\Rightarrow$ (a) follows from the proof of Theorem 3.3. (d) $\Rightarrow(\mathrm{c})$ is Corollary 3.2, and $(\mathrm{d}) \Leftrightarrow(\mathrm{e}) \Leftrightarrow(\mathrm{f})$ is Theorem 3.1. It is also clear that any of (e)-(h) implies (c) since the set before saturation can be taken to be a singleton. Also (a) implies (e)-(h) since the action is continuous. Finally, the direction $(\mathrm{a}) \Rightarrow(\mathrm{i})$ is Theorem 3.4 , and $(\mathrm{i}) \Rightarrow(\mathrm{d})$ is Lemma 2.2 . 


\section{Further REMARKS AND QUESTIONS}

In this final section we discuss further questions arising from our study of the non-extendability property. For some of these questions we do not have complete answers.

Question 4.1. What are the possible complexities of non-extendable sets?

The Becker-Kechris theorem implies that non-extendable sets have to be $\boldsymbol{\Pi}_{1}^{1}$. Our results in the previous sections show that they can be $\boldsymbol{\Pi}_{1}^{1}$-complete, thus of the highest possible complexity, or they can be $\boldsymbol{\Sigma}_{2}^{0}$-complete, the lowest possible complexity if they are not $G_{\delta}$. In fact, this is true among actions for any fixed non-locally-compact Polish group.

The following example demonstrates that, at least for some Polish group actions, the complexity of non-extendable sets can be arbitrary. Let $M$ be an uncountable Polish space and let $P$ be a Polish space satisfying the fact that for any analytic set $A \subseteq M$, there is a closed set $F \subseteq P \times M$ with $A=\pi_{M}(F)$. For example, $P=\mathbb{N}^{\mathbb{N}}$ or $P=\mathbb{R}^{\mathbb{N}}$. Let $G$ be a Polish group and let $b$ be a continuous and transitive action of $G$ on $P$. For example $G=\mathbb{Z}^{\mathbb{N}}$ or $G=\mathbb{R}^{\mathbb{N}}$, respectively, for the above $P$.

Now denote $X=P \times M$. Define a continuous action $a$ on $X$ by

$$
a(g,(p, x))=g \cdot(p, x)=(g \cdot p, x) .
$$

Then $(p, x) E_{a}(q, y)$ if and only if $x=y$. Thus $X$ is a Polish $G$-space and every orbit is closed.

Now fix an analytic set $A \subseteq M$ and a closed set $F \subseteq X$ such that $A=\pi_{M}(F)$. Then $[F]=\pi_{M}^{-1}(A)$. Since $\pi_{M}$ is continuous and $A$ homeomorphic with $[F]_{G} \cap$ $\left\{\left(p_{0}, x\right): x \in M\right\}$, in which $p_{0}$ is a fixed point in $P$, we know that $[F]_{G}$ and $A$ have the same complexity.

We do not know if a similar example exists for an arbitrary non-locally-compact Polish group. Note that in this example the individual orbits are closed (and therefore $F_{\sigma}$ ), thus the non-extendability property cannot be witnessed by an invariant set with only countably many orbits.

This leads us to the concept below and the question that follows.

Definition 4.2. Let $G$ be a Polish group and $X$ a Polish $G$-space. $X$ is said to have the strong non-extendability property if every $G$-orbit in $X$ is $F_{\sigma}$ and $X$ has the non-extendability property.

Question 4.3. Is there a Polish $G$-space with the strong non-extendability property for every non-locally-compact Polish group $G$ ?

We first remark below that the strong non-extendability property need not hold for all Polish $G$-spaces for non-locally-compact Polish groups $G$.

Proposition 4.4. Let $1 \leq p<\infty$ and

$$
\ell^{p}=\left\{x \in \mathbb{R}^{\mathbb{N}}: \sum_{n=0}^{\infty}|x(n)|^{p}<\infty\right\} .
$$

Then with the natual action of $\ell^{p}$ on $\mathbb{R}^{\mathbb{N}}$ the saturation of every $F_{\sigma}$ set is $F_{\sigma}$.

Proof. On the one hand, each $\ell^{p}$ is a $\Sigma_{2}^{0}$ Polishable subgroup of $\mathbb{R}^{\mathbb{N}}$ with the topology induced by the $\ell^{p}$ norm. So the action of $\ell^{p}$ on $\mathbb{R}^{\mathbb{N}}$ is a continuous action of a non-locally-compact Polish group on a Polish space. On the other hand, each $\ell^{p}$ 
is a $K_{\sigma}$ subset of $\mathbb{R}^{\mathbb{N}}$, since for each $M>0$ the set of $x \in \mathbb{R}^{\mathbb{N}}$ with $\|x\|_{p} \leq M$ is a closed subset of the compact set $[-M, M]^{\mathbb{N}}$. To finish the proof it is enough to check that the saturation of every closed set is $F_{\sigma}$. Let $F \subseteq \mathbb{R}^{\mathbb{N}}$ be closed. Then the saturation of $F$ is $\ell^{p}+F$ or a countable union of sets of the form $K+F$ where $K$ is compact. It is easy to see that $K+F$ is closed, and therefore $\ell^{p}+F$ is $F_{\sigma}$.

Similarly one can show the same property if $\mathbb{R}^{\mathbb{N}}$ in the above proposition is replaced by any $\ell^{q}$ for $p<q<\infty$.

Next we remark that if a Polish group admits an action with the strong nonextendability property, then so do its extensions. Let us first formalize the notion of group extensions.

Definition 4.5. A Polish group $H$ extends a Polish group $G$ if there is a closed subgroup $H^{\prime}$ of $H$ and a continuous homomorphism from $H^{\prime}$ onto $G$.

Proposition 4.6. Let $G$ and $H$ be Polish groups such that $H$ extends $G$. If there is a Polish $G$-space $X$ with the strong non-extendability property, then there is a Polish $H$-space $Y$ with the strong non-extendability property.

Proof. Without loss of generality the proposition can be proved in two cases, namely, when $G$ is a factor group of $H$ (i.e., there is a continuous homomorphism from $H$ onto $G$ ) and when $G$ is a closed subgroup of $H$. In the first case any action of $G$ can be trivially lifted to an action of $H$, and one can easily check that the strong non-extendability property is preserved from viewing the underlying space as a $G$-space to the new point of view that the underlying space is an $H$-space.

For the proof of the second case we use the theorem of Mackey and Hjorth (see [1] Theorem 2.3.5) on extensions of Polish group actions. Let $X$ be a Polish $G$-space and let $A$ and $Y$ witness the strong non-extendability property of $X$.

Consider the action of $G$ on $X \times H$ by

$$
g \cdot(x, h)=(g \cdot x, g h)
$$

It is proved in [1] that every orbit is closed and the quotient space is Polish. Let $X^{\prime}=(X \times H) / G$ and let $H$ act on $X^{\prime}$ by

$$
h \cdot[x, k]_{G}=\left[x, k h^{-1}\right]_{G} .
$$

Then $X^{\prime}$ becomes a Polish $H$-space. Moreover, defining $f: X \rightarrow X^{\prime}$ by $f(x)=$ $[x, e]_{G}, f$ is a homeomorphic embedding of $X$ into $X^{\prime}$ with $f(X)$ closed in $X^{\prime}$. Thus if we identify $X$ with $f(X)$ the action of $G$ on $X$ can be identified with an action on $f(X)$. To summarize, the Polish $H$-space $X^{\prime}$ has the properties that $X$ is a closed subset of $X^{\prime}$, the action of $H$ on $X^{\prime}$ extends the action of $G$ on $X$, and every $H$-orbit in $X^{\prime}$ contains exactly one $G$-orbit in $X$.

Now let

and

$$
A^{\prime}=[A]_{H}
$$

$$
Y^{\prime}=Y \cup\left(\left(X^{\prime} \backslash X\right) \cap[\bar{A}]_{H}\right),
$$

where $\bar{A}$ is the closure of $A$ in $X$. It is easy to see that $A^{\prime} \subseteq Y^{\prime}$ since $A \subseteq Y$. We claim that $A^{\prime}$ and $Y^{\prime}$ witness the strong non-extendability of the Polish $H$-space $X^{\prime}$.

First of all $A^{\prime}=(A \times H) / G$ since $A$ is $G$-invariant. Also $A^{\prime}$ is dense in $Y^{\prime}$, since $A$ is dense in $Y$. In fact, we have $[\bar{A}]_{H} \subseteq \overline{A^{\prime}}$, since obviously $\bar{A} \subseteq \overline{A^{\prime}}$ and $\overline{A^{\prime}}=\overline{[A]_{H}}$ is $H$-invariant. 
To see that $Y^{\prime}$ is $G_{\delta}$ in $X^{\prime}$, note that $Y$ is $G_{\delta}$ in $X^{\prime}$ since it is $G_{\delta}$ in $X$ and $X$ is closed in $X^{\prime}$. This also implies that $X^{\prime} \backslash X$ is $G_{\delta}$ since it is open. Finally $[\bar{A}]_{H}=(\bar{A} \times X) / G$ is closed in $X^{\prime}$. Hence $Y^{\prime}$ is $G_{\delta}$ in $X^{\prime}$.

To see that $A^{\prime}$ is not $G_{\delta}$ in $Y^{\prime}$, just note that $A=A^{\prime} \cap X=A^{\prime} \cap Y$ since each $H$-orbit contains exactly one $G$-orbit. So if $A^{\prime}$ was $G_{\delta}$ in $Y^{\prime}, A$ would be $G_{\delta}$ in $Y$ and hence in $X$.

To finish the proof of the non-extendability property, it is enough to show that for any $[x, k]_{G} \notin A^{\prime}$ there is $h \in H$ such that $h \cdot[x, k]_{G} \notin X^{\prime}$. Recall that we have $g \in G$ such that $g \cdot x \notin Y$. So $[g \cdot x, e]_{G}=f(g \cdot x) \in X \backslash Y$. It follows then that $[g \cdot x, e]_{G} \notin Y^{\prime}$. Note that

$$
g k \cdot[x, k]_{G}=\left[x, k(g k)^{-1}\right]_{G}=\left[x, g^{-1}\right]_{G}=[g \cdot x, e]_{G} .
$$

Thus if we let $h=g k$, then $h \cdot[x, k] \notin Y^{\prime}$.

To complete the proof it only remains to check that every $H$-orbit in $X^{\prime}$ is $F_{\sigma}$ if every $G$-orbit in $X$ is $F_{\sigma}$. For this let $x \in X$. Then $X \backslash[x]_{G}$ is a $G$-invariant $G_{\delta}$ subset of $X$, hence a Polish $G$-space. By Hjorth's argument to prove Theorem 2.3.5 of [1], the extension $\left(\left(X \backslash[x]_{G}\right) \times H\right) / G$ is a Polish $H$-space. But it is immediate that this quotient space is homeomorphic and $G$-isomorphic with $Y \backslash[x]_{H}$. Hence $Y \backslash[x]_{H}$ is $G_{\delta}$ and $[x]_{H}$ is $F_{\sigma}$.

As a final remark, we do not know if non-extendable sets in the sense that some action on it cannot be extended necessarily arise in the circumstance described by the non-extendability property.

Question 4.7. If $G$ is a Polish group acting on a subset $A$ of a Polish space $X$ and the action cannot be extended, is there a Polish $G$-space $X^{\prime}$ and a homeomorphic embedding of $X$ into $X^{\prime}$ whose restriction on $A$ is a $G$-embedding?

\section{REFERENCES}

[1] H. Becker and A. S. Kechris, The Descriptive Set Theory of Polish Group Actions, London Mathematical Society Lecture Note Series, vol. 232, Cambridge University Press, 1996. MR.1425877 (98d:54068)

[2] A. S. Kechris, Classical Descriptive Set Theory, Springer-Verlag, Berlin, 1995. MR1321597 (96e:03057)

[3] A. S. Kechris, A. Louveau and W. H. Woodin, The structure of $\sigma$-ideals of compact sets, Trans. Amer. Math. Soc. 301 (1987), no. 1, 263-288. MR0879573 (88f:03042)

[4] M. Lavrentieff, Contribution à la théorie des ensembles homéomorphes, Fund. Math. 6 (1924), 149-160.

School of Mathematical Sciences and LPMC, Nankai University, Tianjin, 300071 , People's Republic of China

E-mail address: dingly@nankai.edu.cn

Department of Mathematics, P.O. Box 311430, University of North Texas, Denton, TEXas 76210

E-mail address: sgao@unt.edu 